\title{
Phase Analysis of Endoatrial Electrograms for 3D Rotor Detection in Atrial Fibrillation
}

\author{
Maddalena Valinoti ${ }^{1}$, Francesca Berto ${ }^{1}$, Martino Alessandrini ${ }^{1}$, Roberto Mantovan ${ }^{2}$, Axel Loewe $^{3}$, \\ Olaf Dössel $^{3}$, Stefano Severi ${ }^{1}$, Cristiana Corsi ${ }^{1}$ \\ ${ }^{1}$ Department of Electrical, Electronic and Information Engineering, Cesena, Italy \\ ${ }^{2}$ Cardiology and Intensive Care Unit, Bufalini Hospital, Cesena, Italy \\ ${ }^{3}$ Institute of Biomedical Engineering, Karlsruhe Institute of Technology, Karlsruhe, Germany
}

\begin{abstract}
Atrial fibrillation $(A F)$ is the most common type of arrhythmia encountered in clinical practice but its maintaining mechanisms remain elusive. Over the last years, various theories have been proposed to target $A F$ mechanisms. Recently, there has been an increasing interest in understanding how spiral waves and rotors sustain AF and how they might be therapeutic targets for catheter-based ablation. Phase mapping has recently been used as a robust method to characterize the spatiotemporal variability of electrical activities. In this study, we propose an independent approach for basket catheter electrogram (EGM) processing to detect rotors in AF. An improved version of the sinusoidal recomposition method for the local activation timings (LATs) has been developed and $3 D$ phase maps have been reconstructed. An algorithm able to detect stable and meandering rotors on the left atrium (LA) surface was then developed. This workflow has been validated on synthetic EGMs and in silico showing excellent results. On in vivo data, we found $4.0 \pm 3.4$ and $4.6 \pm 5.0$ localized and meandering rotors with a persistence in time: $303.2 \pm 58.2 \mathrm{~ms}$ and $302.3 \pm 52.0 \mathrm{~ms}$ respectively.
\end{abstract}

\section{Introduction}

Atrial fibrillation (AF), is the most common type of arrhythmia and a leading cause of hospitalization and death [1]. Unfortunately, its therapy remains suboptimal. Catheter ablation is a non-pharmacological therapy that aims to restore sinus rhythm by eliminating tissue sustaining $\mathrm{AF}$ and is more effective than medications. Haissaguerre et al. [2] revealed that ectopic beats from the pulmonary veins ( $\mathrm{PVs}$ ) may trigger $\mathrm{AF}$, establishing the field of AF ablation with PV isolation as its cornerstone. Although catheter ablation is a promising therapy, its success is limited because the mechanisms that sustain AF, once triggered, are not defined. The success rate of a single procedure in an "optimal" candidate for $\mathrm{AF}$ ablation is between $60 \%$ and $80 \%$. The single procedure in a "less optimal" patient, such as a patient with persistent $\mathrm{AF}$, ranges between $50 \%$ and $70 \%$ and in a patient with continuous $\mathrm{AF}$ is $40 \%$ or less [3]. The FIRM study suggests that human AF is sustained by spiral waves (rotors) and focal sources whose elimination, guided by intra-atrial multi-electrode recordings, may improve the ablation outcome. The "rotor" is a stably rotating pattern (electrical spiral wave) that surrounds a pivot point [4].

The electrical rotor localization is based on phase analysis of the electrograms (EGMs), according to which local activation timings (LATs) are identified as the timing of phase inversion of the signal (i.e transition of its phase from $-\pi$ to $+\pi$ ). In the phase maps, rotors are represented by phase singularity (PS) points [5] that are surrounded by regions in all possible phases of electrical activation [6,7].

The objective of this study was to develop and validate a new approach based on phase analysis of the endoatrial electrograms to detect local activation timings. Phase maps were then reconstructed on the patient-specific LA geometry and, using phase information patterns, stable and meandering electrical rotors were localized and their persistence in time was estimated.

\section{Methods}

\subsection{Data Acquisition}

Six patients with paroxysmal AF, aged 37 to 75 were enrolled in the study at the Bufalini Hospital in Cesena, Italy. All patients underwent an ablation procedure and a 64-pole basket catheter (Constellation, Boston Scientific, Natick, MA, USA) was used to acquire unipolar EGMs in the left atrium (LA).

Digital electro-anatomic atrial shells were created for clinical guidance using the NavX mapping system (St. Jude Medical, Minneapolis, MN, USA). 


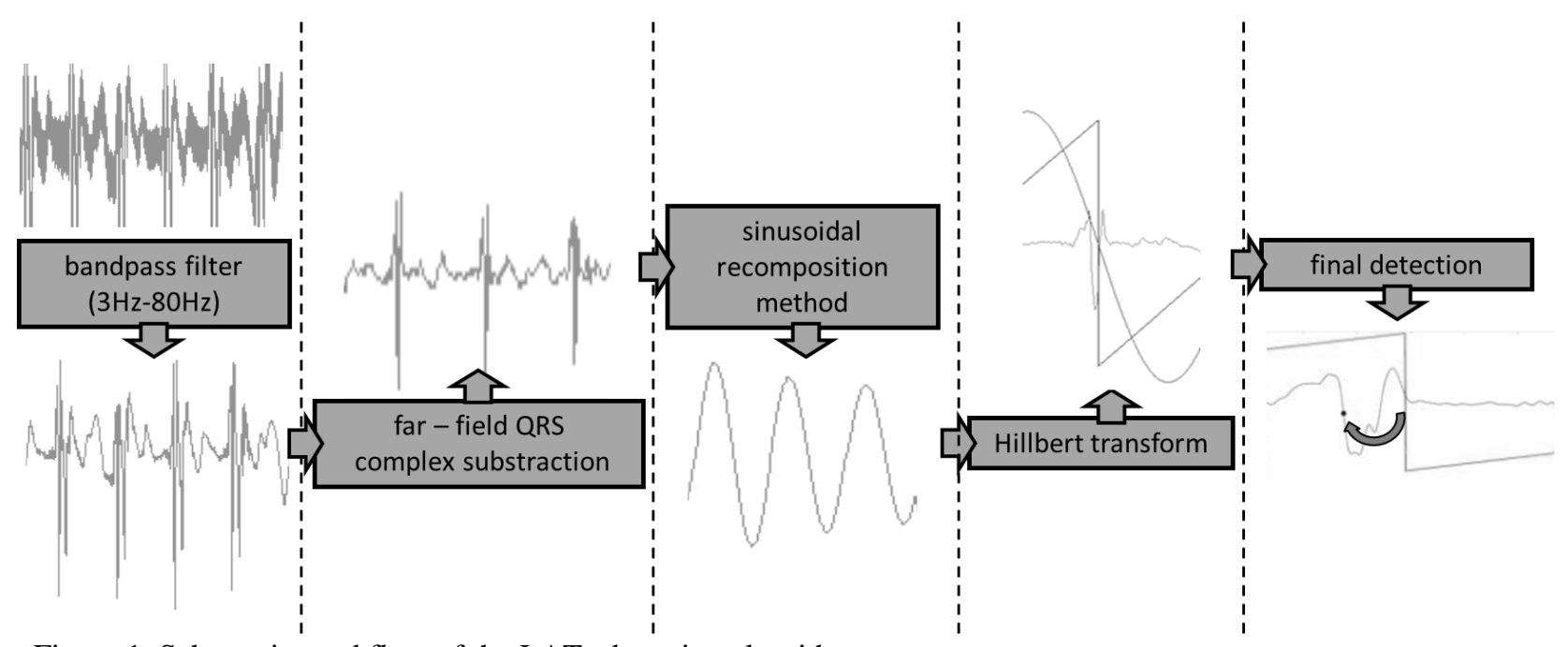

Figure 1. Schematic workflow of the LATs detection algorithm.

\subsection{EGM processing}

A schematic representation of the proposed method is shown in Figure 1.

Since it is well known that the main problem of the basket catheter is its coverage of the endoatrial wall [8], the electrodes whose position was farther than $13 \mathrm{~mm}$ from the LA wall were discarded from the analysis.

The EGMs were band-pass filtered (3 to $80 \mathrm{~Hz}$ ) and farfield QRS complexes were subtracted from the unipolar electrograms by a single-beat cancellation method [9].

The implemented algorithm for LAT detection is a modified version of the electrogram recomposition from sinusoidal wavelets proposed by Kuklik et al. [10]. In the modified sinusoidal recomposition algorithm, the wavelets were only generated in correspondence of a typical morphology of atrial activations; the additional constraints to be satisfied for sinusoidal wavelet generation took into consideration the slope of negative deflections, their amplitude and the duration. The resulting phase inversion points were used to center a window of fixed duration in which the point with the maximum negative derivative corresponded to the LAT.

The 3D phase map was then obtained associating a region of the wall surface to each electrode and mapping the phase signals. The rotor detection algorithm is based on the search of the PS points. A PS is detected if there is a gradual phase transition between neighboring points followed by a phase sign change (red to blue) encompassing the PS point (Figure 2).

The PS is identified as a rotor if its lifespan is greater than one AF cycle length, computed considering the dominant frequency value. In addition, the rotor was defined meandering if the coordinates of its phase singularity point during rotor detection, in a certain time window, moves outside a neighbor greater than $0.5 \mathrm{~cm}$; otherwise the rotor is defined stable.

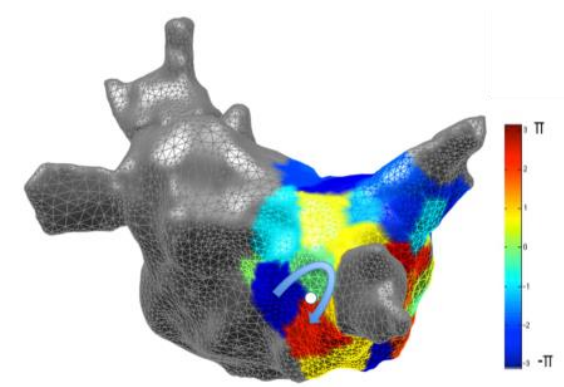

Figure 2. Example of 3D phase map in one frame. The white point represents the detected PS and the arrow indicates the phase transitions.

\subsection{Synthetic EGMs and LA computational model}

To validate LATs, synthetic EGMs were generated as described below starting from LATs manually annotated by two expert electrophysiologists in nineteen measured unipolar EGMs (Figure 3). An atrial EGM can be modelled as the sum of the atrial near field $\left(\mathrm{AA}_{\text {near }}\right)$, the atrial far field $\left(\mathrm{AA}_{\mathrm{far}}\right)$ and the ventricular activity. The ventricular activities and $\mathrm{AA}_{\text {near }}$ were extracted from the measured EGMs. The latter one was extracted considering the electrophysiologists' agreement on LAT detection. $\mathrm{AA}_{\text {far }}$ was obtained employing an autoregressive model [11]. To locate the ventricular activations in $\mathrm{AA}_{\text {far }}$, an atrioventricular node model [12] was applied; a gamma distribution whose parameters were obtained considering the mean of the interval time between the ventricular activations was used for $\mathrm{AA}_{\text {near }}$. To validate the algorithm for the LAAT detection, seven sets of 50 synthetic EGMs 
characterized by an increasing level of noise were generated by modifying the variance values $(\sigma=k$. $\left.\sigma_{M}^{2}, k=0, \mathrm{f}\left(\mathrm{AA}_{\text {near }}\right), 0.1,0.2,0.5,0.7,1\right)$ of the white noise serving as input to the autoregressive model.

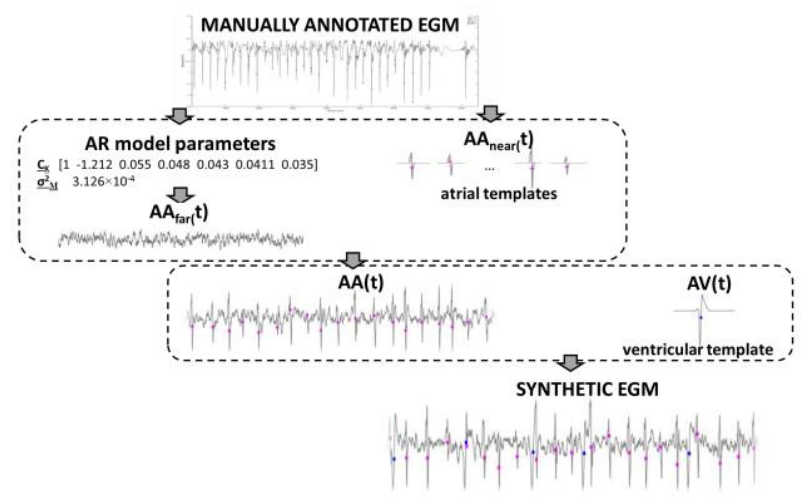

Figure 3. Workflow applied to generate synthetic EGMs from manually annotated EGMs

A computational model of LA electrophysiology was used as benchmark for the rotor detection algorithm validation. The 3D LA anatomy was derived from preoperative MR. The ionic currents were computed using the Courtemanche model suitably modified to account for spatial heterogeneities [13], fiber orientation [14] and AF induced remodeling [15]. Electrical propagation was computed by the monodomain solver acCELLerate [13]. The 64 unipolar EGMs of a simulated basket catheter were computed. A stable reentry was induced by applying a premature stimulus. Two simulations with different catheter positions were performed.

\section{Results}

\subsection{Validation results}

The comparison between the detected LATs on synthetic EGMs with the ground truth atrial activations $\left(\mathrm{AA}_{\text {near }}\right)$ is reported in the Table 1; a detection was considered successful if it matched the $\mathrm{AA}_{\text {near }}$ within a window of $50 \mathrm{~ms}$. In Figure 4, an example of a synthetic EGM is shown.

The performance of the proposed method was compared with the ones presented in [10] on the same set of 350 synthetic EGMs. The two techniques reported similar value of PPV and Se for low levels of noise, up to $k=0.1$; the PPV and sensitivity of the sinusoidal recomposition method were systematically lower up to $10 \%$.

- Preliminary results on rotor detection validation are shown in Figure 5. When the rotor was poorly covered, the algorithm was not able to detect the simulated rotor on the surface (top panels). After catheter repositioning, the simulated rotor was correctly identified (bottom panels).
Table 1. Quantitative results of LATs detection on synthetic EGMs for increasing levels of noise. TA: true activations; $\mathrm{EA}$ : estimated activations; $\mathrm{TP}=$ true positive; $\mathrm{FP} / \mathrm{N}=$ false positive/negative; $\mathrm{PPV}=$ positive predictive value; Se: sensitivity.

\begin{tabular}{cccccccc}
\hline$\#$ & TA & EA & TP & FP & FN & $\begin{array}{c}\text { PPV } \\
(\%)\end{array}$ & $\begin{array}{c}\text { Se } \\
(\%)\end{array}$ \\
\hline 1 & 2000 & 1958 & 1893 & 65 & 107 & 97 & 95 \\
2 & 2049 & 2054 & 1892 & 162 & 157 & 92 & 92 \\
3 & 2048 & 2000 & 1832 & 168 & 216 & 92 & 89 \\
4 & 2000 & 2058 & 1765 & 293 & 235 & 86 & 88 \\
5 & 2049 & 2128 & 1629 & 499 & 421 & 77 & 79 \\
6 & 2000 & 2084 & 1399 & 685 & 605 & 67 & 70 \\
7 & 2000 & 2110 & 1254 & 852 & 742 & 60 & 63 \\
\hline
\end{tabular}

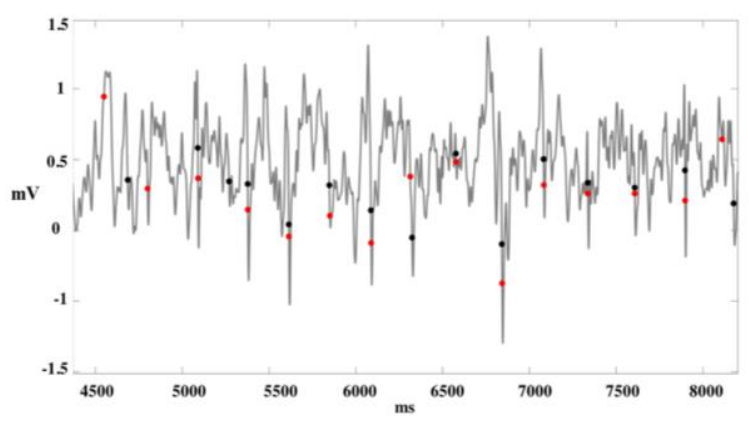

Figure 4 Example of a synthetic EGM with the highest level of noise added. Black dots: detected LATs; red dots: timings of $\mathrm{AA}_{\text {near }}$.

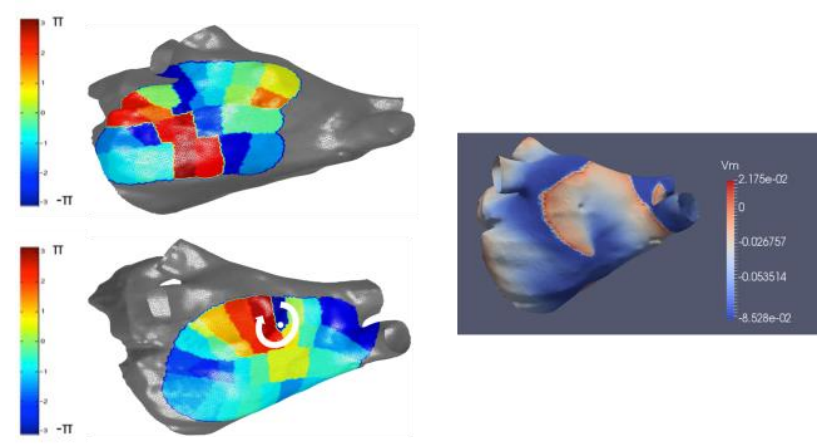

Figure 5. Example of 3D phase maps (left panels) obtained using the simulated EGMs and the computational model (right panels).

\subsection{Results on in vivo EGMs}

Considering $13 \mathrm{~mm}$ as maximum distance between the electrodes and the atrial wall, the total number of processed in vivo EGMs signals was 348 .

The average coverage of the basket catheter was: $43.3 \pm 7.8 \%$. 
In the six patients, on average $4.0 \pm 3.4$ stable rotors and 4.6 \pm 5.0 meandering rotors with a persistence in time: 303.2 $\pm 58.2 \mathrm{~ms}$ and $302.3 \pm 52.0 \mathrm{~ms}$ respectively, were identified.

An example of a PS point identified as a rotor is shown in the Figure 6.

All along the recordings, the rotors were preferentially detected in the PV neighbourhood (4 patients) or in the LA roof (1 patient), whereas they were not concentrated in specific sites in 1 patient.

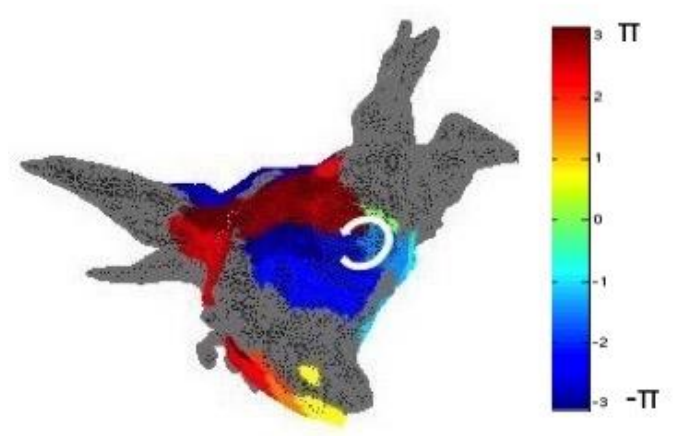

Figure.6 Example of a PS identified as a stable rotor on the 3D surface.

\section{Conclusion}

Nowadays rotor identification and ablation is one of the more debated topics in the electrophysiological community. Phase analysis is considered a robust method for identifying and quantifying spatiotemporal organization of AF. This study proposes an improved method for signal phase reconstruction for atrial activation timing and rotor detection. Due to the very low agreement between LATs manual annotation performed by two experienced electrophysiologists, the validation of the proposed approach on synthetic EGMs was mandatory. Validation results shows the performance remains accurate also in presence of a high level of noise. Rotor detection is based on the PS concept and preliminary comparison with the rotating waves generated by the computational model showed correct identification of PS points highlighting the importance of the basket coverage for reliable identification.

Our independently developed phase mapping method demonstrated effective in the analysis of the basket catheter data. Preliminary results show the presence of rotors in LA, preferentially in PV sites. Rotor spatial stability and temporal persistence needs further investigation. The developed technique, once validated on a larger number of patients, may provide a tool for investigating the effects of different catheter coverage, designing the number of electrodes and intra-spline distance for optimal LA surface coverage aiming at LAT detection and rotor identification.

\section{References}

[1] Miyasaka Y, Barnes ME, Gersh BJ, et al. Secular trends in incidence of atrial fibrillation, Circulation 2006;114:119-25.

[2] Haissaguerre M, Jais P, Shah DC, et al. Spontaneous initiation of atrial fibrillation bt ectopic beats originating in the pulmonary veins. N Engl J Med 1998;339:659-66.

[3] Calkins H. Catheter Ablation to Maintain Sinus Rhythm. Circulation 2012;125:1439-45.

[4] Narayan SM, Krummen DE, Shivkumar K, et al. Treatment of Atrial Fibrillation by the Ablation of Localized Sources CONFIRM, Trial, JAAC, 2012; 60: 628-36.

[5] Lee YS, Song JS, Hwang M. et al. A New Efficient Method for Detecting Phase Singularity in Cardiac Fibrillation, PLoS One, 2016; 11(12): 1-14.

[6] Kuklik P, Zeemering S, van Hunnik A. et al. Identification of rotors during Human Atrial Fibrillation Using Comtact Mapping and Phase Singularity Detection: Technical Considerations. IEEE Trans Biomed Eng, 2017:64(2):310318.

[7] Rottmann M, Unger L, Kaltenbacher W. et al. Methods for analyzing signal characteristics of stable and unstable rotors in a realistic heart model, Computing in cardiology 2015, 42:485-488, https://doi.org/10.1109/CIC.2015.7410953.

[8] Oesterlein T, Frisch D, Loewe A. et al. Basket-Type Catheters: Diagnostic Pitfalls Caused by Deformation and Limited Coverage, BioMed Research International, 2016, doi. 10.1155/2016/5340574.

[9] Ng J, Kadish AH, Goldberger J.J. et al. Technical Consideration for Dominant Frequency Analysis, J Cardiovasc Electrophysiol, 2007;18:757-764.

[10] Kuklik P, Zeemering S, Maesen B, et al. Reconstruction of Instantaneous Phase of Unipolar Atrial Contact Electrogram Using a Concept of Sinusoidal Recomposition and Hilbert Transform, IEE Trans Biomed Eng 2015;62:296-302.

[11] Rivolta MW, Mainardi LT, Sassi R. Synthetic Atrial Electrogram Generator, in Proc. of XIII Mediterranean Conference on Medical and Biological Engineering and Computing, IFMBE 2013;14: 670-73.

[12] Corino VDA, Sandberg F, Lombardi F. et al. An Atrioventricular Node Model for Analysis of the Ventricular Response During Atrial Fibrillation, IEEE Trans Biomed Eng, 2011;58:3386-95.

[13] Krueger MW, Dorn A, Keller DUJ, et al. In silico modeling of atrial repolarization in normal and atrial fibrillation remodeled state. Med Bio Eng Comput 2013;51:1105-19.

[14] Wachter A, Loewe A, Krueger MW, et al. Mesh structureindependent modeling of patient-specific atrial fiber orientation, Current Directions in Biomedical Engineering, ISSN 2364-5504, https://doi.org/10.1515/cdbme-2015-0099.

[15] Loewe A, Wilhelms M, Dössel O, et al. Influence of chronic atrial fibrillation induced remodeling in a computational electrophysiological model. Biomedical Engineering Biomedizinische Technik 59 (2014): S929-S932.

Address for correspondence.

Maddalena Valinoti

DEI, University of Bologna,

Viale Risorgimento 2, 40136, Bologna, Italy

maddalena.valinoti2@unibo.it 